\title{
¿EXISTE DIFERENCIA EN EL LENGUAJE DE LOS NIÑOS SEGÚN SU NIVEL SOCIAL? REVISIÓN Y DISCUSIÓN DEL TEMA
}

\author{
Verónica Aliaga Prieto* \\ Marcia Poblete Ríos ${ }^{\text {t* }}$ \\ Vilma Fenieux Honores ${ }^{* * * t}$
}

\begin{abstract}
RESUMEN
La siguiente revisión bibliográfica permitió a las autoras reflexionar con respecto a las posibles razones que influyen negativamente en un adecuado dominio lingüístico, en niños provenientes de sectores socioeconómicos y socioculturales deprivados de Chile. Se discute la relación existente entre el manejo del lenguaje y el éxito o fracaso en los procesos de aprendizaje que experimentan los estudiantes durante sus primeros años de educación básica, y la importancia de propiciar ambientes que favorezcan un desarrollo lingüístico correcto. Realizada la revisión, se concluye que el medio social y cultural que circunde al niño, así como también la estimulación del desarrollo del léxico y la riqueza de las experiencias a las que esté sometido, son factores de gran importancia en la adquisición del lenguaje, beneficiando o perjudicando al individuo en su desempeño social y profesional futuro.
\end{abstract}

Palabras clave: deprivación, nivel sociocultural, lenguaje, habilidades de aprendizaje.

\section{IS THERE A DIFFERENCE IN THE LANGUAGE OF CHILDREN ACCORDING TO THEIR SOCIAL LEVEL? REVIEW AND DISCUSSION OF THE TOPIC}

\begin{abstract}
The following bibliographic review allowed the authors to reflect about the possible reasons that exert a negative influence on the appropriate linguistic competence of children coming from deprived economic and socio-cultural sectors in Chile. The existing relationship between language competence and the success or failure in the students' learning process, during their first years of primary education, to the importance of providing students with suitable environments intended to foster the development of appropriate linguistic competence, is discussed. It was concluded then, that the surrounding socio-cultural environment, as well as the stimulation of their lexical development, together with the richness of their experiences, are very important factors for language acquisition. It will benefit or endanger the individuals in their social and professional future performance.
\end{abstract}

Keywords: deprivation, sociocultural level, language competence, learning skills

* Profesora de Educación Diferencial, Magíster en Ciencias de la Educación. Departamento de Humanidades, Universidad de Atacama, Copiapó, Chile. veronica.aliaga@uda.cl

* Licenciada en Educación, Profesora de Inglés, Psicopedagoga y Magíster en TEFL. Instituto de Idiomas, Universidad de Atacama, Copiapó, Chile. marcia.poblete@uda.cl

Profesora de Castellano y Filosofía, Psicopedagoga. Liceo de Música de Copiapó, Chile. vifh72@, hotmail.com 
¿Existe diferencia en el lenguaje de los niños según su nivel social? Revisión y discusión del tema - Verónica Aliaga, Marcia Poblete y Vilma Fenieux

\section{Introducción}

El trabajo que a continuación se presenta es una reflexión que da cuenta de la relación existente entre los distintos niveles socioeconómicos y socioculturales nacionales y el manejo lingüístico que muestran los niños que crecen en dichos ambientes. Al mismo tiempo, se reflexiona sobre la relación existente entre el dominio lingüístico de los individuos; el éxito o fracaso de los mismos en los procesos de aprendizaje en los que se ven involucrados; y el beneficio futuro de un manejo lingüístico socialmente aceptado.

Los objetivos que se persiguen con este artículo son:

a. Conocer y comprender las razones debido a las cuales existe un bajo nivel de competencia lingüística en algunas personas provenientes de estratos socioeconómicos y socioculturales deprivados.

b. Reflexionar respecto de la relación entre el manejo del lenguaje y las dificultades o facilidades en el logro de aprendizajes de los alumnos.

c. Reflexionar respecto de la importancia de la estimulación temprana en la adquisición del lenguaje y el beneficio futuro de un dominio lingüístico correcto.

Habiendo reflexionado sobre los aspectos indicados y luego de respaldar los supuestos sostenidos por las investigadoras con literatura especializada, fue posible concluir que sí importa el medio social y cultural que circunde al niño y sí importa cuánto se le hable y cuánto se estimule el desarrollo del léxico y la calidad de las experiencias a las que esté sometido. Está en nosotros abrirle las puertas al niño. Aquellas puertas del aprendizaje que estarán más abiertas mientras mayores sean sus capacidades lingüísticas. Es probable también que el lenguaje que un individuo utilice, evidencie la pertenencia a un determinado estrato sociocultural, lo que, en caso de ser correcto y educado, podría resultar beneficioso; pero en caso contrario, podría resultar perjudicial e impedir una óptima integración social y desempeño profesional. 


\section{Marco Teórico}

\section{Diagnóstico y crítica de la situación actual}

En la ciudad de Copiapó como también en otras ciudades del país, existe gran cantidad de escuelas municipales que trabajan con alumnos en situación de riesgo social, esto se refiere a:

- niños provenientes de hogares disfuncionales

- situación económica precaria, lo que en muchos casos obliga a los niños a efectuar trabajos esporádicos para aportar al ingreso familiar

- baja estimulación en el hogar

- pocas o nulas condiciones que propicien el estudio

- carencias afectivas

- padres escasamente instruidos o con estudios incompletos

Si esto es como se describe podemos, suponer entonces, que los alumnos asisten a la escuela con gran irregularidad y escasa motivación, afectando así el desarrollo normal y continuo del proceso de aprendizaje e implicando que los alumnos arrastren vacíos en sus conocimientos que muy probablemente conservan hasta su estado adulto. Por vacíos en este caso particular, nos referiremos a faltas relativas a una deficiente competencia lingüística, que sería indicador de la cercanía a un entorno culturalmente deprivado. Es así que resulta interesante despertar el interés temprano por mejorar las competencias lingüísticas en los estudiantes, con el propósito de poder aminorar la desventaja social que implica provenir de un nivel socioeconómico y cultural limitado, tendiendo a la equidad social.

\section{¿Qué es el Lenguaje?}

El lenguaje es un método exclusivamente humano, le permite al hombre comunicar ideas, estados de ánimo, por medio de un sistema de símbolos producidos de manera deliberada. El lenguaje es una habilidad humana por excelencia, sólo el hombre posee la asombrosa capacidad de comunicarse y de representar la realidad mediante signos convencionales (Sapir, 1956). 
¿Existe diferencia en el lenguaje de los niños según su nivel social? Revisión y discusión del tema - Verónica Aliaga, Marcia Poblete y Vilma Fenieux

Se distingue entre lenguaje verbal y lenguaje no verbal, siendo más estructurado y rico en posibilidades el lenguaje verbal, mientras que el no verbal, se refiere a los gestos, señales, etc., que acompañan al mensaje formal.

Considerando al lenguaje como un instrumento de comunicación, revisaremos entonces este último término. La comunicación puede considerarse como un proceso o acción, la acción misma de comunicar o también como el contenido que se transmite, lo comunicado, el mensaje.

Al hablar de comunicación, se pone énfasis en las nociones de emisión y recepción de información, utilizando términos como emisor, receptor, canal, etc.; que son los elementos más usados en lingüística y psicología.

Considerando la comunicación en el ámbito de la psicología del lenguaje podemos definirla así: "intercambio de información o puesta en común de significaciones, intencionadas en una relación humana determinada”. (Bermeosolo, 1999).

Como se ha dicho, el lenguaje es el que permite la comunicación entre los seres humanos; por lenguaje nos referiremos al hablado y al escrito.

\section{Adquisición del Lenguaje}

El desarrollo del lenguaje, comienza mucho antes de la primera palabra que el niño dice a finales de su primer año.

Los psicolingüistas estiman que los niños, independientemente de su idioma, pasan por un itinerario universal de etapas en la adquisición del lenguaje; por supuesto que existen diferencias entre niño y niño, pero hay patrones generales evolutivos; incluso en situaciones adversas para la adquisición del lenguaje verbal, igualmente se desarrolla alguna forma del lenguaje. 
Para realizar un análisis que resulte más comprensible, se dividirá esta adquisición de la lengua en sus componentes de desarrollo fonológico, morfosintáctico, semántico y pragmático, pero sin olvidar que existe una interacción entre todos los niveles y que este es un fenómeno que se da como un todo.

El desarrollo tiende a comenzar con la sílaba base que marca el máximo contraste consonante-vocal: Consonante con máximo cierre y vocal con máxima apertura (por ejemplo: "pa”), añadiéndose paulatinamente los demás.

Hay sonidos que el niño ya ha articulado en la etapa del balbuceo, pero que con dificultad logra producir dentro de las palabras; se trata de sonidos que deben ser articulados ahora dentro de un sistema, en secuencias predeterminadas, y no al azar como antes.

Hacia los tres años y medio el niño puede ser entendido con bastante facilidad, pero tan sólo cuando ingresa a la escuela, está en condiciones de dominar casi todos los sonidos de la lengua con sus combinaciones (diptongos, grupos consonánticos, etc.).

\section{Desarrollo del Lenguaje}

\section{Desarrollo morfosintáctico}

El proceso de construcción del sistema de la lengua, expresado en el desarrollo morfosintáctico, ha sido visto desde variadas perspectivas y diferentes criterios.

Varios autores distinguen estadios, centrándose en el Promedio de longitud de los enunciados (MLU, en inglés Mean length of utterance) del niño, este lmpv (en español, longitud media de producción verbal) (Rondal, 1978) aumenta hasta aproximadamente los 6 años; luego el lenguaje puede disminuir en longitud, pero se hace más complejo. 
¿Existe diferencia en el lenguaje de los niños según su nivel social? Revisión y discusión del tema - Verónica Aliaga, Marcia Poblete y Vilma Fenieux

Otros investigadores utilizan criterios preferenciales de los progresos en la elaboración de determinadas estructuras sintácticas: sintagmas nominales, sintagmas verbales, adquisición de la negación o de la interrogación, morfemas gramaticales, tiempos verbales, verbos irregulares, tipos de oraciones, etc.

Los diversos autores que se han ocupado del desarrollo morfosintáctico muestran algunas coincidencias en la delimitación y caracterización de las etapas iniciales: "Etapa de una palabra", "Etapa de dos palabras", etc. O etapa de los I2 a I8 meses, etc.

\section{Desarrollo semántico y pragmático}

Una vez realizado el desarrollo morfosintáctico, es posible darse cuenta lo difícil que es separar la sintaxis de la semántica, considerando que los progresos en un aspecto dependen y se entrelazan con los correspondientes en el otro, al determinar estructuras, también se determinan significados.

El contenido y la expresión (significado y significante) se van interpolando a medida que avanza el léxico gramatical.

Las palabras del niño de cinco o seis años suelen tener un campo muy amplio de significado, ya que para expresar la diversidad y complejidad de situaciones que viven poseen relativamente pocos términos, y así, cada significante debe servir para hacer referencia a varios significados.

Una de las características del léxico de esta edad (seis, siete años) es la creación de palabras inventadas a partir de la analogía que practica la lengua, de acuerdo con una lógica significativa para el niño, por ejemplo, futgol, por fútbol.

\section{Lenguaje y Aprendizaje}

La entrada a la escuela trae un enriquecimiento importante del léxico del niño, tanto cualitativa como cuantitativamente. 
Desde cada área del conocimiento el niño va adquiriendo el léxico de cada contexto cultural en que se mueve (matemáticas, comprensión del medio, relatos de cuentos, etc.). En esta captación intuitiva, que puede ser reforzada por la reflexión del adulto, el niño se prepara para adquirir no sólo el código lingüístico específico para comprender temas de su interés, sino los diferentes códigos (gestual, parsonimia, etc.) que va a necesitar para las diferentes actividades que deberá enfrentar.

La calidad y el nivel de desarrollo lingüístico que un individuo alcance, influirá de manera determinante, no sólo en el diario vivir, sino también en la posibilidad de incorporar nuevos aprendizajes a su vida y además, a la forma de interacción con el medio ambiente.

Existen muchas manifestaciones de un desarrollo psicolingüístico deficiente, originadas como consecuencia de la pobreza, carencias afectivas familiares o falta de incentivos para aprender, que no necesariamente corresponden a una patología individual aún cuando predispongan para el fracaso escolar. De este modo, no sorprende que algunos autores que han estudiado el manejo de las competencias lingüísticas en grupos de estudiantes, hayan encontrado que las dificultades que se presentan en el procesamiento del lenguaje influyen de manera negativa en el proceso lector y, por ende, en la asimilación de aprendizajes (Busch, 1980 y Liberman, I982, citados por Garton, 1994).

Muchos niños que ingresan al sistema escolar y que han sido criados en zonas marginales -rurales o urbanas- presentan dificultad en la adaptación y en los procesos de aprendizaje, probablemente no por la presencia de factores clínicos, sino por la falta de estimulación que ha retrasado de alguna manera la maduración de sus procesos cognitivos. A partir de esto y de las investigaciones anteriormente expuestas, se puede concluir que las condiciones familiares y sociales que se determinan por el hecho de una carencia de recursos, ya sea determinado por las carencias económicas o por una pobreza de hábitos culturales, conlleva que en un individuo, a pesar de tener todas las capacidades y competencias para desarrollar 
¿Existe diferencia en el lenguaje de los niños según su nivel social? Revisión y discusión del tema - Verónica Aliaga, Marcia Poblete y Vilma Fenieux

el lenguaje, este se ve mermado, así como también se pueden generar déficit cognitivos que se ven reflejados en el aprendizaje. Por otra parte, todos los problemas que implica el no tener un lenguaje desarrollado y adecuado para distintas situaciones de carácter formal o cotidiano, pueden desencadenar una serie de restricciones en las capacidades académicas, lingüísticas y sociales del individuo. Por lo tanto, "con un mínimo de capital lingüístico, una persona quedará excluida del mercado comunicativo. Quien dispone de menos palabras genera una inseguridad en su desempeño lingüístico, lo que desemboca en una gran desigualdad social” (Peñaloza et al., 2008).

El lenguaje marginal constituye, entonces, una barrera no sólo frente a nuevos aprendizajes, sino, además, un problema permanente en las relaciones interpersonales. La Psicóloga educativa, Mariana Miras Mestres (I982), considera que el contexto escolar, lejos de ser neutro, privilegia un determinado tipo de discurso y consiguientemente una determinada orientación, que Bernstein identifica como perteneciente a las clases altas. Una incidencia importante que tiene la clase social y la evolución del lenguaje que esta conlleve, influye en el rendimiento escolar, ya que una precaria adquisición de lenguaje y escasa estimulación de los procesos cognitivos llevan a lo se conoce como fracaso escolar. Miras (1982), se refiere a las dependencias del lenguaje como las formas de lenguaje, que dependen de características culturales y no de características individuales, determinan la posibilidad o, al contrario, la imposibilidad de adquirir aquellas competencias -a la vez intelectuales y sociales- cuya posesión condiciona el éxito escolar y profesional.

Una característica de nuestra cultura es la creciente distancia psicológica y social que existe entre niños de diferentes estratos socioeconómicos. Para tener una prueba de ello, resulta suficiente revisar y comparar los resultados obtenidos a nivel nacional de enseñanza básica de distinto nivel económico o de distinto sector geográfico, analizando los resultados de las pruebas SIMCE realizadas por el MINEDUC a los cuartos años básicos del país. 
El psicólogo Luis Bravo Valdivieso (1999) afirma: "existen diferencias entre niños pertenecientes a distintos medios socioeconómicos y culturales en algunos procesos cognitivos y verbales asociados a los trastornos para aprender a leer".

\section{El Desarrollo del Lenguaje Oral y su Relación con el Aprendizaje de la Lectura}

El sistema escolar en sí mismo crea una problemática compleja al niño que debe adaptarse a nuevas tareas, personas y normas y responder a lo que se espera de él. Es en este sistema escolar donde, además de muchas otras habilidades, las habilidades lingüísticas del niño se ponen a prueba para acceder a la lectura y la escritura.

No existe ningún otro aspecto del aprendizaje que incida tan directamente en la adquisición general de éstos que la lectura y la escritura, por tanto, el éxito o el fracaso en dicha tarea son decisivos para el niño y su ajuste posterior. No es casual que la mayor incidencia de problemas de aprendizaje en la etapa escolar inicial sean los que tienen relación con la lectura y la escritura.

\section{¿Qué necesita un niño para aprender a leer?}

A continuación, consideraremos algunas habilidades primordiales que se requieren para desarrollar armónicamente el desarrollo lector:

Semánticas: que son habilidades para usar el conocimiento, conceptos familiares para comprender el significado individual de palabras escritas; y Fonológicas: que son habilidades para unir, segmentar y analizar los sonidos del habla, aprender los nombres y los sonidos de las letras del alfabeto, aprender a formar palabras.

No sólo las anteriores están en la base del aprendizaje lector, también el niño necesita habilidades sintácticas y gramaticales, que implican habilidad para dividir las oraciones en frases, de manera de facilitar la comprensión, para usar el conocimiento implícito de las partes de la oración para entender: tiempo, número, entre otras y para juzgar 
¿Existe diferencia en el lenguaje de los niños según su nivel social? Revisión y discusión del tema - Verónica Aliaga, Marcia Poblete y Vilma Fenieux

si una oración está gramaticalmente bien estructurada como ayuda para el reconocimiento de palabras y para anticipar palabras.

Factor importante en cuanto a todo aprendizaje es la Comprensión, la que implica reconocimiento de palabras individuales y acceso al significado de ésta. La comprensión del significado depende de: la facilidad con la que el niño puede reconocer las palabras de un texto; también el desarrollo del lenguaje, por ejemplo, desarrollo del vocabulario, conocimientos de gramática y sintaxis, entre otras.

Con el lenguaje oral, se relacionan especialmente: la "Conciencia Fonémica", que es parte de un sistema de habilidades más amplias, que se denominan conciencia fonológica. Esta es la habilidad para reconocer y usar todos los tipos de unidades de sonidos, tales como: fonemas, sílabas, palabras.

De acuerdo a lo anterior, un estudiante que tiene problemas de procesamiento fonológico es quien tiene dificultad para percibir, decodificar, recordar, organizar y recuperar información verbal y tendrá dificultades en la asociación correcta entre las representaciones de las letras y el lenguaje hablado.

Existen varios estudios posteriores, que han confirmado que existe una estrecha relación entre la conciencia fonémica y la habilidad lectora, no sólo en los primeros años, sino a través de todos los años de la escolaridad (Bravo, 1995).

\section{Lenguaje y Sociedad}

Dada su naturaleza social, el ser humano permanentemente experimenta la necesidad de comunicarse ya sea consigo mismo o con sus semejantes. Esta comunicación debe ser de calidad, así lo exige nuestra cambiante y multifacética sociedad moderna.

El lenguaje humano constituye un sistema complejo de comunicación que nos permite expresar nuestros estados de conciencia y cristalizar nuestros pensamientos. Su desarrollo es continuo y se afirma con 
la interacción que cada individuo logre hacer con los otros y con su entorno. Por ello, se puede afirmar que el lenguaje "constituye la primera huella de la sociedad sobre el pensamiento individual, que a su vez potencia la posibilidad de establecer posteriores mensajes con sus semejantes" (Rey, 1976).

Es probable que el nivel de desarrollo lingüístico que presente cada individuo, tenga una estrecha relación con el medio en que éste crece y se desenvuelve. De alguna manera nosotros somos el reflejo de nuestro entorno. Seremos imitadores de nuestros padres, amigos, vecinos, etc.

Sin duda, el desarrollo que logremos sobre las competencias lingüísticas, se encuentra relacionado con la presencia que en mayor o en menor grado tengan en nuestra vida elementos como: las satisfacciones materiales, el prestigio, el amor propio, entre otros.

Cada grupo social posee "tipos lingüísticos" que ofrecer a sus integrantes. Modelos de expresión diferentes al resto, tanto en lo cualitativo como en lo cuantitativo (Musen et al., 1998).

Los seres humanos somos, de alguna manera, herederos de los indicadores lingüísticos que caracterizan al medio en el cual nos desenvolvemos y desarrollamos.

Esta evidencia es tan fuerte que por ella podemos ser identificados claramente como pertenecientes a tal o cual clase sociocultural, grupo etario, localidad geográfica, etc. El aprendizaje de estos indicadores, que caracterizan nuestro desempeño lingüístico, es en un alto porcentaje por imitación, y esto, preferentemente de la primera fuente de influencia; la familia, el barrio, los amigos, por nombrar algunos. Si el modelo lingüístico es elevado, correcto, amplio y variado, el individuo poseerá un lenguaje de similares características; si por el contrario, el modelo resulta limitado y gramaticalmente incorrecto, el comportamiento lingüístico dará evidencia de ello. Peña (1988), considera que el proceso de aprendizaje lingǘstico en el ser humano, se da de manera activa mediante la 
¿Existe diferencia en el lenguaje de los niños según su nivel social? Revisión y discusión del tema - Verónica Aliaga, Marcia Poblete y Vilma Fenieux

interacción con su entorno, de esta manera el sujeto va aprendiendo las formas, contenido y usos lingüísticos propios del grupo social en el cual se desarrolla (Peña, 1998).

Lo anterior constituye el correlato de lo afirmado por Albert Bandura, quien sostiene que gran parte de lo que el niño aprende es el resultado de la observación y de la imitación de la conducta presentada por un modelo, en muchos casos sin la presencia de reforzamiento. Tan importante resulta el medio en el desarrollo de elementos lingüísticos como el habla, que un niño puede arrastrar incluso, las características articulatorias de sus padres (citado en Musen et al., 1998).

Podríamos entonces decir, que en gran parte de los casos un uso lingüístico deficiente tiene relación con el hecho de haber crecido en un medio social, cultural y lingüísticamente deprivado. Chomsky, sostiene que los seres humanos estamos lingüísticamente programados para desarrollar el lenguaje, pero es el entorno quien debe detonar la emergencia de esta posibilidad de aprendizaje (citado en Mercer, 1987). En este sentido, la educación formal tiene mucho que aportar, dado que a partir de la repetición del repertorio de signos y la implementación con técnicas, se puede llegar a activar aquella zona que permita a nuestros niños un mejor horizonte lingüístico.

\section{Las Diferencias Lingüísticas según la Clase Social}

La teoría más conocida en lo que respecta a las diferencias de empleo del lenguaje entre clases sociales y su incidencia en la escolaridad es la del sociólogo Berstein (1975). Dentro de la teoría de Bernstein, que es una teoría sociológica, interesa también a la psicolingüística y a la psicología social, entre otras; dada la implicancia que tiene para la mejor realización de este trabajo, consideraremos lo que se refiere a la psicolingüística.

Según Bernstein, los niños de clase baja utilizan un código lingüístico restringido, con relación al que aprenden los niños de clase mediaalta; estos últimos aprenden un código elaborado. 
Los códigos restringido y elaborado se caracterizan por una serie de aspectos que se pueden reagrupar en tres categorías: los aspectos psicolingüísticos, sociolingüísticos y cognitivos. Es la primera categoría la que a continuación se describirá:

$I^{\circ}$ El código restringido contiene una gran proporción de frases cortas simples, con una predominancia de construcciones activas, declarativas e imperativas. En cambio, el código elaborado pone en juego una sintaxis precisa y más compleja.

$2^{\circ}$ En el código elaborado, las relaciones entre los acontecimientos referidos, son especificadas con ayuda de toda la gama de medios formales propuestos por la gramática de la lengua; la selección de los adjetivos y de los adverbios, se realiza a partir de un amplio y matizado abanico. En el código restringido, se evidencia más bien un empleo rígido y estereotipado de las conjunciones, de los adjetivos y de los adverbios más comunes.

$3^{\circ}$ El uso de pronombres impersonales es más raro en el código restringido que en el código elaborado.

$4^{\circ}$ El código restringido contiene un gran número de expresiones hechas y triviales. El código elaborado en cambio tiene secuencias que muestran un compromiso personal del hablante en las opciones emitidas, por ejemplo: "En mi opinión", "creo que".

$5^{\circ}$ El código restringido es un lenguaje de significaciones implícitas. La significación de los mensajes es verbalmente más explícita en el código elaborado.

Esta teoría prevé que, en ciertas situaciones, los usuarios habituales del código restringido pueden utilizar el código elaborado y viceversa. Lo que diferencia las clases sociales desde el punto de vista lingüístico, es la frecuencia de utilización de un código dado en los principales contextos de socialización.

A pesar de esta teoría y otras investigaciones revisadas (Schriner y Miner, 1968; Jenkinson y Weymouth, 1976), no es posible afirmar que las diferencias observadas corresponden a déficits o a simples diferencias de funcionamiento psicolingüístico. Ningún código es intrínsecamente superior a otro, ambos están adaptados a los medios sociales en los que emergen y se mantienen. 
Otra investigación a cargo de Basil Berstain, sociólogo inglés de la década del '60, logró aislar "códigos lingüísticos restringidos y códigos complejos o mensajes”. Los primeros, utilizados por las clases económicamente deprimidas, dieron cuenta que la primera fuente de socialización, es decir, la madre, interactúa con sus hijos sobre la base de oraciones breves, sencillas, gramaticalmente simples y fáciles de entender, que se refieren primordialmente a cosas concretas y acontecimientos del aquí y ahora. En cambio, las madres de clase media, utilizan un código complejo, donde el lenguaje es un medio para socializar, disciplinar, transmitir normas morales, comunicar sentimientos y emociones. En consecuencia, el niño de clase media cuenta con una plataforma lingüística que le facilitará llegar a abstraer, generalizar, relacionar, etc. Mientras tanto, el niño de nivel económico deprivado contará con un código que le dificultará su desarrollo, dado que se encuentra muy ligado a lo concreto, con un contexto determinado y que no trasciende (Musen et al., 1998).

\section{Conclusión}

El niño adquiere lo que será su lenguaje y su habla a través, podríamos decir, de dos vertientes; de su relación con otros, o sea, desde lo social y desde lo biológico, dadas las capacidades cognitivas que posee. Todo niño adquiere lenguaje, pero es desde lo social, en su aprender con otros donde verdaderamente se desarrolla este lenguaje, muchas investigaciones se realizan y se han realizado hacia este respecto, no todas son concordantes, pero desde nuestro punto de vista, sí importa el medio social y cultural que circunde al niño y sí importa cuánto se le hable y cuánto se estimule el desarrollo del léxico y la calidad de las experiencias a las que esté sometido.

El lenguaje sigue siendo un tema interesante de estudiar y describir. Como se decía en el texto, saber más de lenguaje es saber más acerca del hombre. Los profesores tenemos la gran misión de educar en el lenguaje y se constituye precisamente el uso del lenguaje en la gran herramienta que permitirá la adquisición del resto de los aprendizajes. Está en nosotros abrirle las puertas al niño, esas puertas 
del aprendizaje estarán más abiertas mientras mayores sean sus capacidades lingüísticas.

Es probable también que el lenguaje que un individuo utilice evidencie la pertenencia a un determinado estrato sociocultural, lo que, en caso de ser correcto y educado, podría resultar beneficioso; pero, en caso contrario, podría resultar perjudicial. Es decir, sobre todo si consideramos, que es con ese lenguaje con lo que el individuo deberá enfrentar a la sociedad y al medio laboral o profesional en el que se desempeñe. Sin lugar a dudas, la brecha social se estrecha frente al desarrollo de las competencias verbales que el niño posea. Esto nos permite reafirmar la importancia de una temprana estimulación en el desarrollo del lenguaje, lo que podría motivar la elaboración de un plan de mejoramiento del lenguaje, aplicable a niños y niñas provenientes de estratos sociales deprivados de nuestro país.

\section{Bibliografía}

Ausubel, D. y Sullivan, E. (1983). "El desarrollo infantil III - Aspectos Lingüísticos, Cognitivos y Físicos”. Desarrollo del lenguaje: capítulo $l$. Ediciones Paidós. Barcelona, España.

Bermeosolo, J. (1999). "Psicología del lenguaje". Apuntes para educadores y estudiantes de pedagogia. Ediciones P.U.C. Santiago, Chile.

Bermeosolo, J. (1990). "Habilidades Psicolingüísticas y Procesamiento de la Lengua escrita en el Disléxico". Boletín de Investigación de la Facultad de Educación. Pontificia Universidad Católica de Chile. № 8, pág. I3-24. Santiago.

Bermeosolo, J. (1999). "Trastornos del Aprendizaje y Problemas del Aprendizaje" Encuentro Nacional: Dificultades, Diagnóstico y Tratamiento en Educación Especial. Universidad Católica del Maule. Talca.

Boada, H. (1986). El desarrollo de la comunicación en el niño. Editorial Anthropos. Barcelona, España.

Crystal, D. (198I). Lenguaje infantil, aprendizaje y linguística. Editorial Médica y Técnica. Barcelona, España.

Dale, P. (1980). Desarrollo del lenguaje. Editorial Trillas. México.

Fernández, A.; Ferrades y J. Sarramora (1982). Didáctica del Lenguaje. Ceac. S.A. España. 
¿Existe diferencia en el lenguaje de los niños según su nivel social? Revisión y discusión del tema - Verónica Aliaga, Marcia Poblete y Vilma Fenieux

Fernández, S. Conquista del lenguaje en preescolar y ciclo preparatorio.

Flavell, J. (1979). La psicología de Jean Piaget. Editorial Paidós. Buenos Aires, Argentina.

Garton, A. (1994). Interacción social y desarrollo del lenguaje y la cognición. Editorial Paidós. Barcelona.

Herriot, P. (1977). Introducción a la Psicología del Lenguaje. Editorial Labor. Barcelona.

Ingram, D. (1989). First Language Adquisition: Method, Description and Explanation. Cambridge: Cambridge University Press.

Labinowicz, E. (1982). Introducción a Piaget, Pensamiento, Aprendizaje, Enseñanza. Ediciones Fondo Educativo Interamericano. México.

Macshane, J. (I99I). Cognitive Development. Editorial T.J. Press. Britain, England.

Mercer, C. (1987). Dificultades del Aprendizaje II. Ceac. S.A. España.

Miras, M. M. (1982). Psicología Educativa, "La problemática social lenguaje-Clase social: Revisión de algunos aspectos”. Pág. 51 y 52.

Monfort, M. (1995). El niño que babla. Editorial Cepe. Madrid, España.

Musen, P.; J. Janeway y J. Kagan (I998). Desarrollo de la Personalidad del Niño. Trillas, México (p. 563).

Olerón, P. (198I). El niño y la adquisición del lenguaje. Editorial Morata. Madrid, España.

Papalia, D. y Wendkos, S. (I997). Desarrollo bumano, con aportaciones para Iberoamérica. Editorial McGraw Hill, sexta edición. Bogotá, Colombia.

Peña, J. C. (1998). Manual de logopedia. Barcelona. Masson S.A. Pág. I8.

Rey, J. (1976). Cultura y Mensaje. Pablo del Río, Madrid.

Rondal, J. (1982). El desarrollo del lenguaje. Editorial Médica y Técnica. Madrid, España.

Siguán, M. (1984). Estudios sobre psicología del lenguaje infantil. Editorial Pirámide. Madrid, España.

Vygostky, L. (1973). Pensamiento y Lenguaje. Cap. 2. Editorial Pléyade. Buenos Aires, Argentina. 